\title{
Estimation of the Enhancement Factor for Mercury in Air
}

\author{
Allan H. Harvey
}

Received: 26 January 2010 / Accepted: 9 March 2010 / Published online: 31 March 2010

(C) US Government 2010

\begin{abstract}
A combination of classical thermodynamics and molecular theory was used to calculate the enhancement factor (ratio of vapor-phase partial pressure to saturated vapor pressure) for liquid mercury in equilibrium with air at standard atmospheric pressure at temperatures from $0{ }^{\circ} \mathrm{C}$ to $40^{\circ} \mathrm{C}$. The enhancement factors range from 1.0025 at $0{ }^{\circ} \mathrm{C}$ to 1.0016 at $40^{\circ} \mathrm{C}$. This enhancement is too small in magnitude, and in the wrong direction, to explain a difference that has been noted between equilibrium vapor concentrations implied by the vapor pressure of pure mercury and those assumed in some calibration procedures.
\end{abstract}

Keywords Air · Calibration · Enhancement factor · Mercury · Nonideality · Second virial coefficient · Vapor concentration

\section{Introduction}

A major source of mercury pollution is atmospheric emissions from combustion of coal. Instruments to measure mercury in air must be calibrated; this is challenging because of the low concentrations involved. A recent analysis [1] estimated an expanded relative uncertainty (coverage factor $k=2$ ) of $17 \%$ for these measurements, with much of the uncertainty coming from uncertainty in the concentration of mercury in air used in calibration.

Contribution of the National Institute of Standards and Technology, not subject to copyright in the United States.

\footnotetext{
A. H. Harvey $(\varangle)$

Thermophysical Properties Division, National Institute of Standards and Technology, 325 Broadway, Boulder, CO 80305-3337, USA

e-mail: aharvey@boulder.nist.gov
} 
These calibrations use devices that attempt to produce a saturated concentration of mercury in air at a known temperature, with the mercury-containing air sampled by syringe for injection into the analytical instrument. The first extensive analysis of this procedure was performed by Dumarey et al. [2]. In conjunction with the work, a correlation was developed (although not actually given in [2]) for the concentration of mercury in air as a function of temperature. This correlation is often called the Dumarey equation and is commonly used [1,3-5] to estimate the vapor concentration of mercury produced by such an apparatus.

From a thermodynamic standpoint, the saturated vapor concentration must be closely related to the vapor pressure. Huber et al. [6] performed an extensive analysis of experimental vapor pressures and other thermodynamic data, producing a correlation for the vapor pressure of mercury that they estimated to have an expanded relative uncertainty (coverage factor $k=2$ ) of $1 \%$ at near-ambient temperatures. This correlation has subsequently been confirmed by a single vapor-pressure datum near $21^{\circ} \mathrm{C}$ [7] and by high-accuracy experiments at temperatures above $200{ }^{\circ} \mathrm{C}$ [8].

However, the saturated vapor concentration of mercury predicted from the vaporpressure correlation of Huber et al. [6] (assuming mercury vapor is an ideal gas; this assumption will be discussed in Sect. 4.3) does not agree with the concentrations predicted by the Dumarey equation. This discrepancy is shown graphically by Brown et al. [1], with the concentrations based on the vapor pressure approximately $7 \%$ higher than those from the Dumarey equation. This disagreement is much larger than the $1 \%$ uncertainty of the vapor-pressure correlation (the uncertainty of the Dumarey equation is unclear, but the reproducibility of the related calibration procedure seems to be about $1 \%$ [1,2]). Brown et al. [3] performed a gravimetric experiment that in principle could have resolved the discrepancy, but their uncertainties were large enough (approximately 13\%) that both the results fall within their error bars.

The prediction of concentration based on vapor pressure alone does not describe the mercury saturation devices exactly, because the devices contact mercury with air, while the vapor-pressure equation describes an air-free system. While use of a saturated air stream could produce differences for a variety of reasons (such as incomplete saturation, sampling problems, or chemical reaction), here we restrict our analysis to thermodynamic effects. It is well known that gases enhance the vapor concentration of a solute above its condensed phase; this is an important concept in humidity metrology [9] and in supercritical fluid extraction [10]. The "enhancement factor" is due primarily to the deviation of the vapor from ideal-gas behavior and to the effect of pressure on the chemical potential of the condensed phase.

Before proceeding, we note that this thermodynamic effect cannot explain the discrepancy noted above. As reflected in its name, the enhancement factor for physically realistic systems increases the vapor concentration of the solute above what would be calculated based on its vapor pressure alone. This effect is in the opposite direction to that needed to explain the difference between the Dumarey equation and the vaporpressure correlation. Nevertheless, it is valuable to quantify the enhancement factor to understand the thermodynamic effect of air on the vaporization of mercury. If and when the discrepancy is resolved, accurate knowledge of the enhancement factor will reduce the uncertainty in the characterization of any device that produces saturated mercury in air. 
In this article, we first outline the thermodynamic description of the enhancement factor. In Sect. 3, we compute the portion due to the effect of pressure on the condensed phase (known as the Poynting correction). In Sect. 4, we compute the effect of vapor-phase nonideality. We then combine these and present calculated values for the enhancement factor and vapor concentration, and close with some discussion of the results. All results in this article are for air at standard atmospheric pressure $(101.325 \mathrm{kPa})$, but the thermodynamic framework can be used at other pressures in a straightforward manner.

\section{Thermodynamic Background}

The enhancement factor $E$ for a condensed-phase solute (here designated component 2 ) in a pressurized gas is defined [10] as the ratio

$$
E(T, p)=\frac{y_{2} p}{p_{2}^{\mathrm{s}}(T)},
$$

where $p_{2}^{\mathrm{s}}$ is the vapor pressure of the pure solute and $y_{2}$ is the solute vapor mole fraction at thermodynamic equilibrium between the gas and the condensed phase at pressure $p$ and absolute temperature $T$. $E$ is the ratio of the partial pressure of the solute in the vapor to its equilibrium vapor pressure at $T$; the amount by which $E$ exceeds unity is a measure of how much the gas enhances the vaporization of the solute.

If we neglect the extremely tiny solubility of air in liquid mercury, equating the fugacities of the solute in the condensed and vapor phases produces the following expression for the enhancement factor:

$$
E=\frac{\phi_{2}^{\mathrm{s}}}{\phi_{2}} \exp \left(\int_{p_{2}^{\mathrm{s}}}^{p} \frac{v_{2}}{R T} \mathrm{~d} p\right),
$$

where $\phi$ is the fugacity coefficient (the ratio of the fugacity to that in an ideal-gas mixture). The superscript $\mathrm{s}$ in $\phi_{2}^{\mathrm{s}}$ designates the pure-component saturation state (where the pressure is $p_{2}^{\mathrm{s}}$ ), while $\phi_{2}$ in the denominator is the fugacity coefficient in the gas mixture at pressure $p . v_{2}$ is the molar volume of the pure condensed phase, and $R$ is the molar gas constant.

Because the vapor pressure of mercury at our conditions of interest is very small (less than $1 \mathrm{~Pa}$ at $25^{\circ} \mathrm{C}$ ), the saturated vapor will be very nearly an ideal gas, and we can set $\phi_{2}^{\mathrm{s}}=1$ (the relative error in this assumption is on the order of $10^{-7}$; see Sect. 4.3). Furthermore, since $p$ is not large (101.325 kPa in our calculations), the small compressibility of liquid mercury can be neglected, and we can use its molar volume at standard atmospheric pressure throughout the range of integration in Eq. 2. With these two simplifications, Eq. 2 becomes

$$
E=\frac{\exp \left(\frac{\left(p-p_{2}^{\mathrm{s}}\right) v_{2}}{R T}\right)}{\phi_{2}} .
$$


The numerator in Eq. 3 is known as the Poynting correction, while the denominator represents the departure of the vapor-phase fugacity from that of an ideal gas.

\section{Poynting Correction}

The numerator in Eq. 3 requires the molar volume of liquid mercury, which is known very accurately at near-ambient conditions. We use the expression recommended by Bettin and Fehlauer [11] for the mass density at standard atmospheric pressure:

$$
\rho_{\mathrm{Hg}}(T)=\frac{\rho_{20}}{1+A_{0}(t-20)+A_{1}(t-20)^{2}+A_{2}(t-20)^{3}+A_{3}(t-20)^{4}},
$$

where $\rho_{20}$ is $13545.850 \mathrm{~kg} \cdot \mathrm{m}^{-3}$, the recommended density at standard atmospheric pressure and $20^{\circ} \mathrm{C}, t$ is the Celsius temperature, $A_{0}=1.811891 \times 10^{-4}, A_{1}=$ $7.5669 \times 10^{-9}, A_{2}=3.6094 \times 10^{-11}$, and $A_{3}=1.5502 \times 10^{-14}$. The atomic mass of $200.59 \mathrm{~g} \cdot \mathrm{mol}^{-1}$ [12] is used to convert the mass density to a molar volume.

The Poynting correction also requires the molar gas constant $R=8.314472$ $\mathrm{J} \cdot \mathrm{mol}^{-1} \cdot \mathrm{K}^{-1}[13]$ and the vapor pressure $p_{2}^{\mathrm{s}}(T)$, for which we use the correlation of Huber et al. [6]. Any error in $p_{2}^{\mathrm{s}}$ will have a negligible impact on Eq. 3, because $p_{2}^{\mathrm{s}} \ll p$.

\section{Fugacity Coefficient}

\subsection{Theory}

The fugacity coefficient of a component in a mixture is rigorously related to a composition derivative of the Helmholtz energy [10]. For low and moderate pressures, it is adequate and accurate to represent the thermodynamics by the virial expansion, which is a series of corrections to ideal-gas behavior:

$$
z \equiv \frac{p v}{R T}=1+\frac{B(T)}{v}+\frac{C(T)}{v^{2}}+\cdots
$$

where $v$ is the molar volume and $B, C$, etc. are virial coefficients that depend only on temperature and (for a mixture) on composition.

The composition dependence of the virial coefficients is rigorously given by statistical mechanics. For the second virial coefficient $B$, the relationship for a mixture of $m$ components is

$$
B_{\text {mix }}(T)=\sum_{i=1}^{m} \sum_{j=1}^{m} y_{i} y_{j} B_{i j}(T),
$$

where $B_{i j}$ at a given temperature depends only on the interaction between one molecule of species $i$ and one molecule of species $j$. 
For the relatively low pressures of interest here, Eq. 5 can be truncated after the first correction. After some thermodynamic manipulations, this allows the fugacity coefficients to be computed as [10]

$$
\ln \phi_{i}=\left(2 \sum_{j=1}^{m} y_{j} B_{i j}-B_{\text {mix }}\right) \frac{p}{R T} .
$$

For the mercury-air system, Eq. 7 becomes

$$
\ln \phi_{\mathrm{Hg}}=\left(y_{\mathrm{Hg}}\left(2-y_{\mathrm{Hg}}\right) B_{\mathrm{HgHg}}+2 y_{\mathrm{a}}^{2} B_{\mathrm{a}, \mathrm{Hg}}-y_{\mathrm{a}}^{2} B_{\mathrm{aa}}\right) \frac{p}{R T},
$$

where subscripts " $\mathrm{Hg}$ " and "a" designate mercury and air, respectively. While air is really a mixture, we can follow the example of the humidity community $[9,14]$ and treat air as a pseudo-pure component without altering the thermodynamics.

From Eq. $8, \phi_{\mathrm{Hg}}$ can be computed at any temperature and pressure if the appropriate $B_{i j}$ are known. Equation 8 also requires the composition, which is initially unknown. Because the mole fraction of mercury is small, an effective procedure is to first assume $y_{\mathrm{Hg}}=0$, use Eq. 8 to compute an initial guess for $\phi_{\mathrm{Hg}}$, and then use Eqs. 3 and 1 to compute a new $y_{\mathrm{Hg}}$ for use in Eq. 8 to produce a final value of $\phi_{\mathrm{Hg}}$ for calculating the final enhancement factor from Eq. 3. This successive substitution procedure could be repeated, but in practice a single iteration is sufficient for convergence to mutually consistent values of $\phi_{\mathrm{Hg}}, y_{\mathrm{Hg}}$, and $E$.

\subsection{Second Virial Coefficient for Air-Air}

The second virial coefficient for air at near-ambient temperatures has been extensively studied. We use the formula recommended by Hyland and Wexler [9]:

$$
B_{\mathrm{aa}} /\left(1 \mathrm{~cm}^{3} \cdot \mathrm{mol}^{-1}\right)=b_{0}+b_{1} / T+b_{2} / T^{2}+b_{3} / T^{3}
$$

where $b_{0}=34.9568, b_{1}=-6687.72 \mathrm{~K}, b_{2}=-2.10141 \times 10^{6} \mathrm{~K}^{2}$, and $b_{3}=$ $9.24746 \times 10^{7} \mathrm{~K}^{3}$. Values of $B_{\mathrm{aa}}$ at temperatures of interest for this work are shown in Table 1.

Table 1 Second virial coefficients (in $\mathrm{cm}^{3} \cdot \mathrm{mol}^{-1}$ ) for the mercury-air system

\begin{tabular}{lllllll}
\hline$t\left({ }^{\circ} \mathrm{C}\right)$ & $B_{\mathrm{aa}}$ & $B_{\mathrm{HgHg}}$ & $B_{\mathrm{N}_{2}, \mathrm{Hg}}$ & $B_{\mathrm{O}_{2}, \mathrm{Hg}}$ & $B_{\mathrm{Ar}, \mathrm{Hg}}$ & $B_{\mathrm{a}, \mathrm{Hg}}$ \\
\hline 0 & -13.15 & -502 & -25.9 & -31.5 & -76 & -27.6 \\
10 & -10.80 & -468 & -22.6 & -28.5 & -71 & -24.3 \\
20 & -8.64 & -438 & -19.5 & -25.7 & -67 & -21.3 \\
25 & -7.62 & -424 & -18.1 & -24.4 & -64 & -19.8 \\
30 & -6.65 & -411 & -16.7 & -23.2 & -62 & -18.5 \\
40 & -4.82 & -387 & -14.1 & -20.8 & -58 & -15.9 \\
\hline
\end{tabular}




\subsection{Second Virial Coefficient for $\mathrm{Hg}-\mathrm{Hg}$}

Because $y_{\mathrm{Hg}}$ is small, the term containing $B_{\mathrm{HgHg}}$ in Eq. 8 is negligible for practical purposes. However, we analyze $B_{\mathrm{HgHg}}$ for completeness, because it will introduce some concepts needed for $B_{\mathrm{a}, \mathrm{Hg}}$, and because it illuminates the extent of dimerization (or lack thereof) in mercury vapor.

Experimental measurement of the second virial coefficient for mercury is not practical. However, $B_{\mathrm{HgHg}}$ can be calculated if the pair potential for interacting mercury atoms is known. If quantum corrections are ignored (which is reasonable for heavy atoms such as mercury), the second virial coefficient is rigorously given by [15]

$$
B_{i j}(T)=-2 \pi N_{\mathrm{A}} \int_{0}^{\infty}\left[\exp \left(-U_{i j}(r) / k_{\mathrm{B}} T\right)-1\right] r^{2} \mathrm{~d} r
$$

where $N_{\mathrm{A}}$ is the Avogadro constant, $k_{\mathrm{B}}$ is the Boltzmann constant, and $U_{i j}(r)$ is the potential-energy function between atoms $i$ and $j$ whose centers are separated by distance $r$.

For the function $U_{i j}(r)$, we employ the "CBS/CCSD $(\mathrm{T})+\mathrm{SO}+\Delta \mathrm{T}$ " ab initio potential of Pahl et al. [16], which was developed at a high level of theory with large basis sets and shown to be consistent with the limited experimental study of the dimer. While higher-level calculations at the potential minimum suggest that this potential might be about $3 \%$ too shallow [16], the accuracy is sufficient for our purposes. The resulting values of $B_{\mathrm{HgHg}}$ are shown in Table 1 . These were compared with values computed from two other recent pair potentials [17,18]. Agreement among the values is within $10 \%$, which gives some indication of the uncertainty in our knowledge of the pair potential and therefore in $B_{\mathrm{HgHg}}$.

For pure mercury at saturation, inserting the values of $B_{\mathrm{HgHg}}$ from Table 1 into Eq. 8 with $y_{\mathrm{Hg}}=1$ and $p=p_{2}^{\mathrm{s}}$ yields fugacity coefficients $\phi_{2}^{\mathrm{s}}$ ranging from 0.99999999 at $0{ }^{\circ} \mathrm{C}$ to 0.99999987 at $40^{\circ} \mathrm{C}$. Therefore, our assumption of $\phi_{2}^{\mathrm{s}}=1$ in Sect. 2 is justified. Also, $B_{\mathrm{HgHg}}$ may be converted to an approximate equilibrium constant for the vapor-phase dimerization reaction [10]. At the very low $p_{2}^{\mathrm{s}}$ of interest here, a calculation with the resulting equilibrium constant yields a dimer fraction on the order of $10^{-8}$ to $10^{-7}$, which is of the same magnitude as estimated from mass spectrometric experiments [19]. This shows that vapor-phase dimerization of mercury is negligible at these conditions.

\subsection{Second Virial Coefficient for Air-Hg}

As has been done for the air-water system in humidity standards [14], we can construct $B_{\mathrm{a}, \mathrm{Hg}}$ from the individual contributions of the main components of air:

$$
B_{\mathrm{a}, \mathrm{Hg}}=y_{\mathrm{N}_{2}} B_{\mathrm{N}_{2}, \mathrm{Hg}}+y_{\mathrm{O}_{2}} B_{\mathrm{O}_{2}, \mathrm{Hg}}+y_{\mathrm{Ar}} B_{\mathrm{Ar}, \mathrm{Hg}},
$$


where the mole fractions $y_{\mathrm{N}_{2}}=0.781184, y_{\mathrm{O}_{2}}=0.209480$, and $y_{\mathrm{Ar}}=0.009336$ are obtained from the composition of dry air [20] after subtracting out trace components and renormalizing.

Experimental measurements of the required cross virial coefficients in Eq. 11 are lacking, so again we turn to calculations based on intermolecular potentials.

The simplest of these binaries is the argon-mercury pair, which has a one-dimensional potential. A semiempirical potential has been developed by Sheng et al. [21]. From this potential, Eq. 10 gives values of $B_{\mathrm{Ar}, \mathrm{Hg}}$ as shown in Table 1.

To a good approximation at the temperatures considered here, the nitrogen molecule can be considered a rigid rotor, resulting in a two-dimensional nitrogen-mercury pair potential. This can be described by the distance $r$ between the $\mathrm{Hg}$ atom and the midpoint of the $\mathrm{N}_{2}$ molecule and an angular variable $\theta$, defined as the angle to the $\mathrm{Hg}$ atom as viewed from the $\mathrm{N}_{2}$ midpoint, with $\theta=0$ when the $\mathrm{Hg}$ atom is collinear with the $\mathrm{N}_{2}$ molecule. For angle-dependent potentials, the analog to Eq. 10 is [22]

$$
B_{i j}(T)=-2 \pi N_{\mathrm{A}} \int_{0}^{\infty}\left[\left\langle\exp \left(-U_{i j}(r, \theta) / k_{\mathrm{B}} T\right)\right\rangle-1\right] r^{2} \mathrm{~d} r
$$

where the angle brackets indicate averaging over all mutual orientations at distance $r$. In the two-dimensional coordinate system used here, the number of points on a sphere of radius $r$ at angle $\theta$ is proportional to $\sin \theta$, so the average in this case is taken over all values of $\theta$ with each contribution weighted by $\sin \theta$.

The pair potential of the nitrogen-mercury system has been studied by Ilčin et al. [23] at $0.36 \mathrm{~nm} \leq r \leq 0.7 \mathrm{~nm}$. They developed a three-dimensional potential in which the intramolecular $\mathrm{N}_{2}$ bondlength was allowed to vary, but it can be reduced to a two-dimensional potential by fixing the $\mathrm{N}-\mathrm{N}$ distance at its equilibrium value.

Unfortunately, the potential function of Ilčin et al. [23] extrapolates poorly beyond the range of their ab initio calculations. At short distances, for some values of $\theta$ the potential takes large negative values, when physically it must approach $+\infty$. This can be easily rectified by setting $U_{i j}$ to a large positive number for all distances less than $0.3 \mathrm{~nm}$. At large distances, their $U_{i j}$ does not decay as $r^{-6}$, which theory dictates should be the case for dispersion interactions. This behavior in the "tail" of the potential has a significant influence on $B_{i j}$. We therefore used a revised potential (M. Ilčin, personal communication, 2010) in which additional ab initio calculations at distances beyond $0.7 \mathrm{~nm}$ were used to produce a function with correct long-range behavior. Table 1 shows values of $B_{\mathrm{N}_{2}, \mathrm{Hg}}$ calculated from the revised potential of Ilčin et al. [23] (M. Ilčin, personal communication, 2010).

We are not aware of any study of the pair potential for the oxygen-mercury system. We therefore estimated $B_{\mathrm{O}_{2}, \mathrm{Hg}}$ by scaling the nitrogen-mercury results using an effective Lennard-Jones 12-6 potential. The first step in this procedure was to obtain an effective collision diameter $\sigma_{\mathrm{Hg}}$ for the mercury atom by fixing the Lennard-Jones welldepth $\varepsilon_{\mathrm{Hg}}$ at the minimum of the $\mathrm{Hg}-\mathrm{Hg}$ potential of Pahl et al. [16] $\left(\varepsilon_{\mathrm{Hg}} / k_{\mathrm{B}} \approx 564 \mathrm{~K}\right)$ and finding the value of $\sigma_{\mathrm{Hg}}$ that produced the value of $B_{\mathrm{HgHg}}$ at $20^{\circ} \mathrm{C}$ in Table 1 . This is combined (in the usual linear manner) with the effective Lennard-Jones values of $\sigma$ for $\mathrm{N}_{2}$ and $\mathrm{O}_{2}$ [15] to produce an effective $\sigma$ 
for the $\mathrm{N}_{2}-\mathrm{Hg}$ and $\mathrm{O}_{2}-\mathrm{Hg}$ interactions. The value of $B_{\mathrm{N}_{2}, \mathrm{Hg}}$ at $20^{\circ} \mathrm{C}$ from Table 1 was then used to determine the effective Lennard-Jones $\varepsilon$ for the $\mathrm{N}_{2}-\mathrm{Hg}$ interaction. Finally, an effective $\varepsilon$ for the $\mathrm{O}_{2}-\mathrm{Hg}$ interaction was estimated by scaling the $\mathrm{N}_{2}-\mathrm{Hg}$ value according to the relative values of the effective Lennard-Jones $\varepsilon$ for $\mathrm{N}_{2}$ and $\mathrm{O}_{2}$ given by Hirschfelder et al. [15]:

$$
\varepsilon_{\mathrm{O}_{2}, \mathrm{Hg}}=\varepsilon_{\mathrm{N}_{2}, \mathrm{Hg}}\left(\varepsilon_{\mathrm{O}_{2}} / \varepsilon_{\mathrm{N}_{2}}\right)^{1 / 2}
$$

The effective Lennard-Jones 12-6 potential with $\varepsilon$ from Eq. 13 and $\sigma$ determined by the procedure in the previous paragraph has $\sigma_{\mathrm{O}_{2}, \mathrm{Hg}}=0.350 \mathrm{~nm}$ and $\varepsilon_{\mathrm{O}_{2}, \mathrm{Hg}} / k_{\mathrm{B}}=$ $132.7 \mathrm{~K}$. This was used in Eq. 10 to produce the values of $B_{\mathrm{O}_{2}, \mathrm{Hg}}$ shown in Table 1. While a Lennard-Jones potential is a crude approximation for the $\mathrm{N}_{2}-\mathrm{Hg}$ and $\mathrm{O}_{2}-\mathrm{Hg}$ systems, much of the resulting error in calculating $B_{\mathrm{O}_{2}, \mathrm{Hg}}$ should be canceled out by our constraint that the more rigorously determined values of $B_{\mathrm{N}_{2} \text {, Hg }}$ be reproduced.

With $B_{\mathrm{Ar}, \mathrm{Hg}}, B_{\mathrm{N}_{2}, \mathrm{Hg}}$, and $B_{\mathrm{O}_{2}, \mathrm{Hg}}$ all in place, Eq. 11 can be used to calculate $B_{\mathrm{a}, \mathrm{Hg}}$. The resulting values are shown in Table 1 .

\section{Results}

Table 2 shows the values of the Poynting correction calculated as described in Sect. 3, the fugacity coefficient $\phi_{\mathrm{Hg}}$ calculated from Eq. 8 with the second virial coefficients given in Table 1, and the enhancement factor $E$ calculated from Eq. 3.

Figure 1 shows the enhancement factor as a function of temperature, along with its two components (the Poynting correction and $\phi_{\mathrm{Hg}}$ ). It can be seen that both thermodynamic factors are significant in the calculation of $E$, with the contribution of $\phi_{\mathrm{Hg}}$ two to three times as large as the Poynting correction at these conditions.

It is also of interest to calculate the equilibrium concentration of mercury in air, because this is the quantity used in calibrations. The first step is to calculate the compressibility factor $z$ from Eq. 5 and the known mixture second virial coefficient from Eq. 6. These values of $z$ are shown in Table 2. The vapor concentration of $\mathrm{Hg}$ in $\mathrm{mol} \cdot \mathrm{m}^{-3}$ is then the equilibrium mole fraction of $\mathrm{Hg}$ (computed as described in Sect. 4.1) multiplied by the overall molar density, which is the reciprocal of the molar volume that can be computed from $z$ with Eq. 5. Mass units are obtained by multiply-

Table 2 Thermodynamic quantities for mercury saturated in air at $101.325 \mathrm{kPa}$

\begin{tabular}{lllllll}
\hline$t\left({ }^{\circ} \mathrm{C}\right)$ & $p_{\mathrm{Hg}}^{\mathrm{s}}(\mathrm{Pa})$ & Poynting correction & $\phi_{\mathrm{Hg}}$ & $E$ & $z$ & $c_{\mathrm{Hg}}\left(\mathrm{ng} \cdot \mathrm{cm}^{-3}\right)$ \\
\hline 0 & 0.0270 & 1.000658 & 0.99813 & 1.00253 & 0.999413 & 2.391 \\
10 & 0.0703 & 1.000636 & 0.99838 & 1.00226 & 0.999535 & 6.004 \\
20 & 0.171 & 1.000616 & 0.99859 & 1.00203 & 0.999641 & 14.13 \\
25 & 0.261 & 1.000606 & 0.99869 & 1.00192 & 0.999688 & 21.19 \\
30 & 0.393 & 1.000597 & 0.99878 & 1.00182 & 0.999733 & 31.35 \\
40 & 0.855 & 1.000579 & 0.99895 & 1.00163 & 0.999813 & 65.99 \\
\hline
\end{tabular}




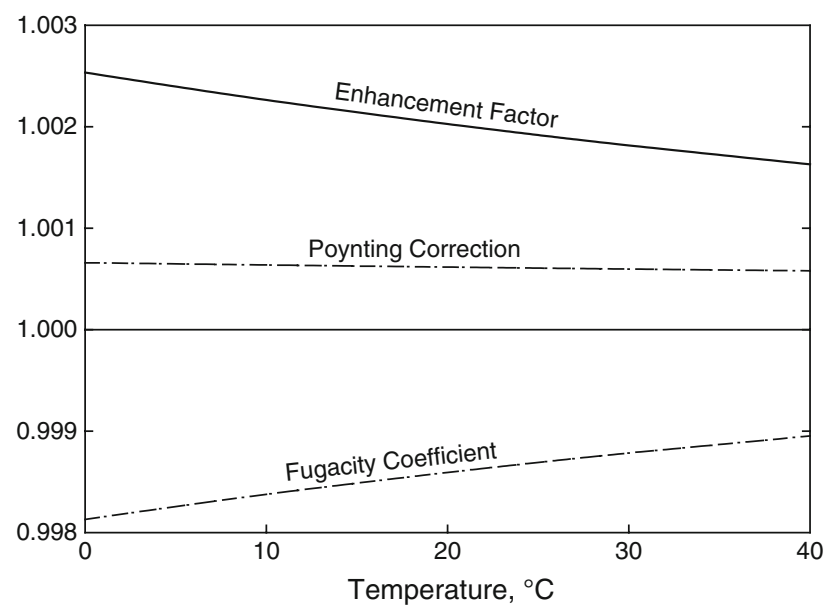

Fig. 1 Enhancement factor $E$, and its two contributing factors as defined in Eq. 3, for liquid mercury equilibrated with air at a pressure of $101.325 \mathrm{kPa}$

ing the molar concentration by $200.59 \mathrm{~g} \cdot \mathrm{mol}^{-1}$. The resulting concentrations $c_{\mathrm{Hg}}$ are shown in the last column of Table 2.

\section{Discussion}

Table 2 and Fig. 1 show that the presence of air at standard atmospheric pressure produces a small, positive thermodynamic enhancement of the equilibrium partial pressure of mercury above its liquid. The enhancement is on the order of $0.2 \%$ at the conditions considered here. In order to produce agreement with the Dumarey equation, a negative enhancement on the order of $7 \%$ would be required, corresponding to $\phi_{\mathrm{Hg}} \approx 1.07$. Such values for vapor-phase fugacity coefficients are physically unrealistic except perhaps at high densities and very high reduced temperatures where repulsive forces dominate, which is not the case here. Any vapor-phase chemical association involving mercury would also reduce its fugacity and lead to $\phi_{\mathrm{Hg}}<1$. It is clear that thermodynamic effects from the presence of air cannot account for the discrepancy noted in Sect. 1.

It can also be noted that the calculated enhancement $(E-1)$ is smaller than the $1 \%$ uncertainty in the vapor-pressure correlation [6]. The uncertainty in the vapor pressure is currently the largest contributor to the uncertainty in a thermodynamic calculation of the saturated vapor concentration of mercury in air.

While the calculations in this work are for dry air, it is not too difficult to incorporate the effect of humidity, which may be present in some practical situations. Humidity will not affect the Poynting correction (which depends only on the pressure), but it will affect the fugacity coefficient $\phi_{\mathrm{Hg}}$ due to the differing vapor composition. The theoretical framework of Sect. 4.1 still applies, but additional second virial coefficients are required. $B(T)$ for pure water [24] and for water with air [14] are taken from the literature. For the water-mercury pair, we apply the same procedure as described 
in Sect. 4.4 for mercury with oxygen. A calculation with $100 \%$ relative humidity at $20^{\circ} \mathrm{C}$ (where our estimate for $B_{\mathrm{H}_{2} \mathrm{O}, \mathrm{Hg}}$ is $-124 \mathrm{~cm}^{3} \cdot \mathrm{mol}^{-1}$ ) yields $\phi_{\mathrm{Hg}}=0.99847$, slightly less than the result for dry air shown in Table 2 . The compressibility factor $z$ is 0.99957 , also slightly less than for dry air. Both of these factors increase the equilibrium vapor concentration compared to that in dry air, but the effect is small, only an incremental $0.02 \%$ increase above the enhancement produced by dry air.

Because the molar volume of mercury is known with high accuracy, the uncertainty in the Poynting correction is negligible. The only significant uncertainty in the calculations reported here is that in $\phi_{\mathrm{Hg}}$, which results almost entirely from uncertainty in $B_{\mathrm{N}_{2}, \mathrm{Hg}}$ and $B_{\mathrm{O}_{2}, \mathrm{Hg}}$. The uncertainties in these second virial coefficients depend on the uncertainties of the pair potentials used to compute them, but no uncertainty estimate was given by Ilčin et al. [23] for their $\mathrm{N}_{2}-\mathrm{Hg}$ potential and the uncertainty of the estimation procedure used for $\mathrm{O}_{2}-\mathrm{Hg}$ is unknown.

Lacking sufficient information for a complete uncertainty analysis, we make some rough estimates. Estimated welldepths for the $\mathrm{Hg}-\mathrm{Hg}$ potential [16-18] vary by about $6 \%$. The potential-energy surface for the $\mathrm{N}_{2}-\mathrm{Hg}$ pair is in some ways easier to calculate from theory because there are fewer electrons, but the extra dimensionality is a complication, and the basis sets and level of theory used by Ilčin et al. [23] (M. Ilčin, personal communication, 2010) were not as advanced as those used in the most recent recent study of the $\mathrm{Hg}-\mathrm{Hg}$ pair [16]. We estimate the $\mathrm{N}_{2}-\mathrm{Hg}$ potential to be uncertain by $10 \%$. Because the $\mathrm{O}_{2}-\mathrm{Hg}$ pair was based on a semiempirical scaling of the $\mathrm{N}_{2}-\mathrm{Hg}$ pair, a reasonable estimate for the uncertainty of that pair potential is $15 \%$ (much of which would be correlated with the $\mathrm{N}_{2}-\mathrm{Hg}$ uncertainty). The effect of these uncertainties in the pair potentials on $B_{\mathrm{N}_{2}, \mathrm{Hg}}$ and $B_{\mathrm{O}_{2}, \mathrm{Hg}}$ can be estimated by perturbing the welldepth $\varepsilon$ in the effective Lennard-Jones calculations described in Sect. 4.4, and these virial coefficients can be propagated into the calculation of $\phi_{\mathrm{Hg}}$. The outcome of this procedure is that the deviation from ideality $\left(1-\phi_{\mathrm{Hg}}\right)$ is uncertain by about $50 \%$, which translates to approximately a $35 \%$ uncertainty in $(E-1)$. It would not be unreasonable to consider this rough estimate to be an expanded uncertainty with coverage factor $k=2$.

This uncertainty analysis depends on the assumption that the $\mathrm{N}_{2}-\mathrm{Hg}$ pair potential of Ilčin et al. [23] is accurate at the level one would expect from the theory used. Compared to the $\mathrm{Hg}-\mathrm{Ar}$ potential, [21] where there is good experimental data, the minimum of the $\mathrm{Hg}-\mathrm{N}_{2}$ potential is about $30 \%$ smaller in magnitude. This is unexpected, since $\mathrm{Ar}$ and $\mathrm{N}_{2}$ have nearly equal polarizabilities. It would be desirable to have independent confirmation of the $\mathrm{Hg}-\mathrm{N}_{2}$ potential, or at least of its welldepth. We note that, if the true $\mathrm{Hg}-\mathrm{N}_{2}$ potential were more similar to the $\mathrm{Hg}-\mathrm{Ar}$ potential, this would make $\phi_{\mathrm{Hg}}$ lower, perhaps by as much as $0.3 \%$ at the conditions considered here. Such a change would increase the discrepancy between the Dumarey equation and the thermodynamic equilibrium vapor concentration by a corresponding amount.

The uncertainty of the calculated enhancement factors could be improved significantly if pair potentials for the $\mathrm{N}_{2}-\mathrm{Hg}$ and $\mathrm{O}_{2}-\mathrm{Hg}$ systems were developed with small, well-documented uncertainties. It should also be possible to measure the enhancement factor (and the vapor pressure) with gravimetric apparatus similar to that recently developed by Meyer et al. [25] for measurement of $\mathrm{H}_{2} \mathrm{O}$ in air in the context of humidity standards. 
Acknowledgments The author thanks his NIST colleagues Marcia Huber, Steve Long, Chris Meyer, and Jay Hendricks for helpful discussions. Michal Ilčin, J. Karl Johnson, Elke Pahl, and Peter Schwerdtfeger supplied helpful information about various pair potentials used in this work.

\section{References}

1. R.J.C. Brown, A.S. Brown, R.E. Yardley, W.T. Corns, P.B. Stockwell, Atmos. Environ. 42, 2504 (2008)

2. R. Dumarey, E. Temmerman, R. Dams, J. Hoste, Analyt. Chim. Acta 170, 337 (1985)

3. A.S. Brown, R.J.C. Brown, W.T. Corns, P.B. Stockwell, Analyst 133, 946 (2008)

4. International Organization for Standardization, ISO 6978-2, Natural Gas-Determination of Mercury_Part 2: Sampling of Mercury by Amalgamation on Gold/Platinum Alloy (ISO, Geneva, 2003)

5. ASTM Standard D-6350-98, Standard Test Method for Mercury Sampling and Analysis in Natural Gas by Atomic Fluorescence Spectroscopy (ASTM International, West Conshohocken, 2003)

6. M.L. Huber, A. Laesecke, D.G. Friend, Ind. Eng. Chem. Res. 45, 7351 (2006)

7. S.E. Long, F. Schaedlich, J.H. Hendricks, Analyst (in preparation)

8. A. Merlone, C. Musacchio, J. Chem. Thermodyn. 42, 38 (2010)

9. R.W. Hyland, A. Wexler, ASHRAE Trans. 89, 520 (1983)

10. J.M. Prausnitz, R.N. Lichtenthaler, E. Gomes de Azevedo, Molecular Thermodynamics of Fluid-Phase Equilibria, 3rd edn. (Prentice Hall, Upper Saddle River, 1999)

11. H. Bettin, H. Fehlauer, Metrologia 41, S16 (2004)

12. M.E. Wieser, J. Phys. Chem. Ref. Data 36, 485 (2007)

13. P.J. Mohr, B.N. Taylor, D.B. Newell, J. Phys. Chem. Ref. Data 37, 1187 (2008)

14. A.H. Harvey, P.H. Huang, Int. J. Thermophys. 28, 556 (2007)

15. J.O. Hirschfelder, C.F. Curtiss, R.B. Bird, Molecular Theory of Gases and Liquids (Wiley, New York, 1954)

16. E. Pahl, D. Figgen, C. Thierfelder, K.A. Peterson, F. Calvo, P. Schwerdtfeger, J. Chem. Phys. 132, $114301(2010)$

17. L.J. Munro, J.K. Johnson, K.D. Jordan, J. Chem. Phys. 114, 5545 (2001)

18. K.T. Tang, J.P. Toennies, Mol. Phys. 106, 1645 (2008)

19. K. Hilpert, J. Chem. Phys. 77, 1425 (1982)

20. A. Picard, R.S. Davis, M. Gläser, K. Fujii, Metrologia 45, 149 (2008)

21. X.W. Sheng, P. Li, K.T. Tang, J. Chem. Phys. 130, 174310 (2009)

22. G.K. Schenter, J. Chem. Phys. 117, 6573 (2002)

23. M. Ilčin, V. Lukeš, L. Bučinský, V. Laurinc, S. Biskupič, Int. J. Quant. Chem. 108, 2150 (2008)

24. A.H. Harvey, E.W. Lemmon, J. Phys. Chem. Ref. Data 33, 369 (2004)

25. C.W. Meyer, J.T. Hodges, R.W. Hyland, G.E. Scace, J. Valencia-Rodriguez, J.R. Whetstone, Metrologia (in press) 